\title{
Complex Text Processing by the Temporal Context Machines
}

\author{
Juyang Weng Fellow, IEEE, Qi Zhang, Mingmin Chi and Xiangyang Xue
}

\begin{abstract}
It is largely unknown how the brain deals with time. Hidden Markov Model (HMM) has a probability based mechanism to deal with time warping, but no effective online method exists that can deal with general active temporal abstraction. By online, we mean that the agent must respond to spatial and temporal context immediately while a sensory stream flows in. By general active temporal context, we mean active (learned) attention selects desirable temporal subsets within a dynamic length of recent history (e.g., beyond bigrams and trigrams). By temporal abstraction, we mean using abstract meaning of context, supervised at the motor end, instead of iconic forms. This paper reports four experiments of complex text processing using the framework of a general-purpose developmental spatiotemporal agent called Temporal Context Machines (TCM), demonstrating its power of forming online, active, abstract, temporal contexts. We show that it perfectly $(100 \%)$ solved a hypothetic problem called New Sentence Problem - after the TCM has learned synonyms under the corresponding contexts, it successfully recognized all possible new sentences (formed from the synonyms) that it has not learned. We show the TCM dealt with the Word Sense Disambiguation Problem where words are ambiguous without context. TCMs were also applied to the Part-of-Speech Problem, where the part of speech of the words in English language is identified according to contexts. In the fourth experiment, TCMs were employed to deal with the challenging Chunking Problem, in which subsequences of words are grouped and classified according to English linguistic units.
\end{abstract}

\section{INTRODUCTION}

$\mathbf{T}$ $\mathrm{HE}$ artificial intelligence community has largely followed a path of hand-designed symbolic representation: Given a task to be executed by a machine, it is the human designer who understands the task and hand-designs the internal concepts (symbols) required by the task before any machine learning can take place. The wave of artificial neural network research in the 80's provided a revolutionary approach: The internal representation of a machine (network) is emergent from incremental learning. The designer specifies the data formats of network input and output as well as the learning mechanisms, but not the actual internal representation. However, the lack of semantic structure in such an emergent representation has restricted artificial neural networks in generating meaningful context representation. We argue that context-dependent internal action known as attention selection is critical for understanding and modeling the autonomous decision process (Buschman \& Miller 2007 [1]) as the architecture proposed in Weng 2007 [2] indicated.

Juyang Weng is with Michigan State University, East Lansing, MI, USA and Fudan University, Shanghai, China (weng@cse.msu.edu). Qi Zhang, Mingmin Chi and Xiangyang Xue are with School of Computer Science, Fudan University, Shanghai, China (email: \{qi_zhang,mmchi,xyxue $\} @$ fudan.edu.cn).
Specifically, this paper addresses a new challenging issue that is of great importance for autonomous development by brain-inspired networks: temporal processing. For clarity, we consider text as the input modality. The input mode we consider here is reading one word at a time and a motor response must be produced at each step. This mode is necessary before studying more sophisticated human reading and enabling machines to read one phrase at a time using visual saccades. When an incrementally learning network reads a document by scanning one word as a time, what kind of representation should be generated immediately online?

At one extreme, every subsequence from the beginning along the text stream of a book corresponds to a different motor action (also a different context state). This type of representation is not very useful as such a state is hardly shared by another experience. At another extreme, every single word corresponds to a different motor action (also a different context state regardless the word preceding it. This type of representation is not powerful either as the machine is only a word-based reflex agent. It cannot make sense from multiple words. In the work presented here, we present a cortex inspired Temporal State Machine that form equivalent spatiotemporal contexts and learn the mapping among these states, so that phrases that correspond to similar meaning are mapped to the same motor output while the internal layer keeps subtle differences. This learned motor representation is used as temporal top-down context for subsequent cortical processing. Based on this scheme, the subset and length of attended temporal context are a result of experience of interactions with the environment (sensory stream and the teacher). For example, "hug me" can be attended as "me" when a child has only learned individual word "me". But, when he has learned "hug me" and the corresponding action, his attended context is not "me," or "hug," but "hug me."

The remainder of this paper is organized as follows: Section II discusses the related prior work. Section III introduces the temporal processing scheme from brain scale, cortex scale, layer scale and neuronal scale. Then, a temporal text processor is described in Section IV. Several experimental results with text data sets are presented in Section V to show its performance. Finally, Section VI provides some concluding remarks.

\section{PRIOR WORK}

Traditionally, text processing is dealt with in a batch fashion: the entire sentence or entire document is received by the agent 
as a batch before the process starts. This includes traditional language models [3] and various computer language compilers and sentence-based interpreters.

There have been many ways for classifying approaches to adaptive and intelligent machines. The most fundamental difference that this paper brings up between the traditional approaches to intelligent and adaptive machines and the brain (also the artificial neural network approach) is whether the internal representation is hand-designed or emergent. We call the former designed-representation approach and the latter emergent representation approach.

\section{A. Symbolic representations}

The HMM [4], [5] is one of the most popular symbolic online temporal model that was meant to deal with time warping. By symbolic, we mean that each node in HMM is an unbreakable symbol.

The meaning of a node in HMM is typically not explicitly determined, but through a pre-processing technique (e.g., kmean clustering) before parameter refinement (e.g., using Baum-Welch algorithm). However, the meaning of each HMM is predefined (e.g., represent a word "Tom") and thus the meaning of each node in the HMM is largely hand-designed. This practice is not possible for autonomous development, since the tasks that the agent will learn are unknown to the programmer before the agent "births" and the internal representation of the agent is not accessible to the human programmer after the agent "births". With autonomous development, for example, we cannot assign a submodel to each particular word, as neither the set of words is known nor the mapping between words to the internal representation.

Many other models, many of which deals with behaviors instead of perception, belong to this category, such as Finite State Machines (FSM), Markov Decision Processes (MDP), Partially Observable MDP (POMDP), Bayesian Networks (also called Semantic Nets and Belief Nets).

\section{B. Distributed representations}

In contrast with symbolic representation with HMM, artificial neural networks uses distributed representation. The brain and artificial neural networks share the same characteristic in representation: the internal representation is emergent from learning experience, regulated by the genes or designed learning mechanisms [6], [7], [8], [9], [10]. An emergent representation is harder for humans to understand, as it is distributed in the sense that a particular meaning is represented by a firing pattern of many neurons and a particular meaning is not uniquely represented by a particular pattern. Thus, an abstract meaning is not represented uniquely by any single neuron but by many patterns that involve multiple neurons. Conversely, a neuron is involved in representing not a single abstract meaning, but many meanings. Because of the distributedness in representation, internal representation of brain cortical regions is largely an open problem.

Certain recurrence in the network is necessary to maintain a sort of state as a representation for temporal context. There have been many forms of recurrent networks. The Elman
Network and the Jordan Network [11] are three-layer networks with context units in the input layer. The Hopfield network [12] is a one-layer recurrent network as a content-addressable memory. The Boltzman machine [13] is a stochastic modification of the Hopfield network trained by a process called simulated annealing. The Long Short-term Memory [14] is for longterm time series predictions. The Echo State Network [15] has a sparsely connected fixed random-weights in the hidden layer, good for reproducing temporal patterns. The wakesleep algorithm by Hinton et al. [16] and later versions [17] have a bottom-up process for recognition and a top-down process for generating inputs. Other major recurrent networks include Wiemer 2003 [18], Roelfsema \& van Ooyen 2005 [19], Sit \& Miikkulainen 2006 [20], Golarai et al. 2007 [21], Reddy et al. 2007 [22], and Weng et al. 2008 [23], etc. The network IHDR has been used to deal with spatiotemporal input sequences to make complex sequential decisions - task transfer learning (Zhang \& Weng 2007) [24]). However, the computational roles of feedback connections in developing meaning-based internal representations have not been clarified in these existing studies.

The recent line of research on autonomous development aims at the goal to enable machines to develop internal representation as attended context and context-dependent action generation through real-time interactions with the environments. Several major advances along this line of research are necessary for the proposed scheme to be scalable to non-trivial sizes:

1) Intermediate bridge representation: Success in the theory of bridge representation by a cortical level: Suppose that a cortical area with response space $Y$ takes bottom-up input space $X$ and top-down input space $Z$. Then, a cortical level self-organizes its neuronal synaptic vectors $\left(\mathbf{v}_{1}, \mathbf{v}_{2}, \ldots, \mathbf{v}_{c}\right)$ so that as a whole they estimate the distribution of the parallel input (product space) of bottom-up and top-down spaces $X \times Z$. In other words, $Y$ is a bridge representation of $X \times Z$. When $Z$ represents abstract meaning, this bridge representation is iconic-abstract (i.e., sensorimotor) [23]

2) Top-down theory and results: Top-down connections are known for long time in neuroscience, but top-down processing has resisted full analysis. As far as we know, we are the only group to have developed a theory for, and demonstrated, class-based internal grouping (i.e., neurons that respond to the same class are nearby) and superior performance with object-based top-down processing [25]. Other groups have described top-down ideas or network properties, e.g., [26].

3) Dual optimality in cortical self-organization: Lobe Component Analysis (LCA) was introduced 2006 [27] and its dual optimality was established recently [28]. It is a neuronal layer model for self-organization, to best address the two pairs of conflicting criteria: (a) spatial: the need for generalization for large size problems with a limited amount neuronal resource; and (b) temporal: fast learning with limited living experience.

Based on these recent advances, we are ready to understand 
and model how the brain deals with time. In terms of the structure of the cerebral cortex, the work here models how temporal context is recursively transformed into a spatial code represented at each neuronal layer as attended context available for the next decision.

Our proposed network model indicates that the response pattern of each function layer corresponds to a (vector) state at this layer. We will show that, unlike the state in POMDP, HMM, Hopfield network and many others, this state integrates information from bottom-up inputs (feature inputs) and topdown inputs (abstract contexts) into a concise continuous vector representation, enabled by the convenient emergent, distributed representation.

\section{Spatiotemporal Abstractive Processing}

In the following section, we explain how abstractive processing models work from different scales. The emphasis is to explain what is brain-inspired abstractive computing and how it is done.

\section{A. The brain is first a developer}

The brain is gradually developed from conception, to birth, to infancy, to adulthood through active sensorimotor experiences. This developmental process depends heavily on interactions among neighboring cells as well as the locally and remotely connected cells, while the entire process is regulated by the genes in the nucleus of every cell [29], [30]. Such interactions determine the representations and functions of different areas of the brain [31], [32], [10], [33]. The brain's internal representation is not innate. It is a compounding result of the genes (which are the same across different cells) and the experience. Thus, instead of modeling the extremely complex brain (end result of development) directly, it is more systematic to model the functional equivalence of its developmental program (i.e., genes) and the process of autonomous brain development, as several researchers have argued [6], [34], [7], [35], [36], [37].

\section{B. Brain scale: Bottom-up top-down sensorimotor pathway model}

1) Abstractive processing from two signal sources: sensor and motor: The brain faces a major challenge. It does not have the luxury of having a human teacher to implant symbols into it, as the brain is not accessible directory to the external human teacher. Thus, it must generate internal representations from the two signal sources: the sensors and the effectors (motors).

Thus, the brain can be noted as a regressor $\mathbf{z}(t)=r(\mathbf{x}(t))$ which maps sensory input $\mathbf{x}(t)$ to motor output $\mathbf{z}(t)$. The twoway connections to the effector enables the brain not only control the effector, but also learn from the actions that are either generated externally (e.g., when a child learns passively while the teacher drags his hand) or internally (e.g., from the reflexes generated by a lower nervous subsystem, such as the spinal cord).
2) The top-down input corresponds to both top-down attention and context: The top-down input reflects the status of the motor (e.g., car or balloon) which enhances the response of the best matched neurons. This information reflects both attention (in terms of enhancing response) and top-down context (in terms of last detected object). Further, the duration of such a top-down context is variable, depending on how long the object in the sensory input has been present and attended.

3) The bottom-up input corresponds to both bottom-up saliency and feature match: The bottom-up input reflects the detected features that have survived competition. The competition includes both goodness of bottom-up match and effect of top-down attention on the earlier layers at the previous time (i.e., they are reflected as bottom-up saliency this time).

\section{Cortex scale: Bottom-up and top-down context folding}

The cerebral cortex contains six layers: layer L1 is the superficial layer and layer L6 is the deep layer. Weng et al. 2008 [23] reasoned that L4 and L2/3 are two feature detection layers with L5 assisting L2/3 and L6 assisting L4, in the sense of enabling long range lateral inhibition. Such long range inhibitions enable different neurons to detect different features. The TCM model was informed by the work of Felleman \& Van Essen [38], Callaway and coworkers [39], [40] and others (e.g., [41]). There are no top-down connections from L2/3 to L4, indicating that L4 uses unsupervised learning (U) while L2/3 uses supervised (S) learning. Weng et al. 2008 [23] reported that such a paired hierarchy USUS led to better recognition rates than the unpaired SSSS alternative. An important function of such paired cortical layer is to enable bottom-up and top-down sources to compete separately, to prevent top-down hallucination.

The motor outputs are often abstract (e.g., communicable meanings are written, said or signed), or can be supervised via learning to give a desired abstract representation. Thus, temporal context can be represented as an abstract meaning at the motor layer, realizing many-to-few mapping - many iconic inputs are mapped to few abstracted motor representations. Furthermore, such an abstracted context at a higher cortex is recursively used as a top-down attention in the middle cortical layer when it receives the next bottom-up sensory input. Such a top-down attention input and the bottomup sensory input conjunctively was recognized (matched) by the corresponding feature detector in the middle layer, which leads to the next desired abstract motor output. This cortical mechanism effectively addresses the well known problem of time warping, that Markovian Models (e.g., Hidden Markov Models (HMM) [4]) intend to address to a limited extent, but without the problem of reduced transitional probability with HMM.

Sequential decisions: In sequential decision making, an outcome depends on multiple decisions in sequence, as each decision depends the outcomes of all the related previous decisions. For example, each response pattern and the motor output at time $t$ depend on the top-down attention signals from the previous motor output at time $t-1$. The TCM recursively makes sequential decisions - generates different 
top-down attentions and bottom-up attention-abstracted features at different times. Each top-down context directs the cortical region (L2/3-L4 combination) to attend to a different part (or feature set) of bottom-up input. This leads to a sequence of different top-down attentions and a sequence of different cortical responses, regardless whether the bottom-up input (or the environment) changes or not. Weng 2007 [2] analyzed that autonomous internal sequential attentions based on emergent representation are sufficient to cause sequential decision making. With this new model, no task-specific internal representations are totally genome specified (or handprogrammed into machines).

\section{Layer scale: Candid Covariance-free Incremental Lobe Component Analysis}

1) Two conflicting criteria and working and long-term memory: The cortical level faces two conflicting criteria: fast adaptation of working memory and large stable long-term memory in the same cortical layer resulted from competition.

2) CCI LCA: dual optimality: The above problem is resolved by distinguishing related memory from unrelated ones. The sparse coding principle (Olshaushen \& Field 1996 [42]) allows only few neurons to fire. Those firing neurons correspond to best matched filters for the current neuronal input and are considered the working memory for the current input. Other neurons in the layer are long-term memory for the current input. Therefore, the role of working memory and long-term memory is dynamic.

Each cortical layer $l$ takes bottom-up input from area $l-1$ and top-down input from area $l+1$ and embeds these two sources of input into its synaptic vectors as a bridge representation between the bottom-up input space and topdown input space.

We have developed a model called Candid Incremental Covariance-free (CCI) LCA [27], [28]). Mathematically, a lobe component is the first principal component of a region in the random input space that the neuron belongs to, assigned through neuronal competition. This model has two (dual) optimalities: (1) Best representation - the smallest average error in the sense of the principal component (the least average error for each neuron to represent any samples in the region represented by the neuron), (2) Best estimation of representation - the smallest average error from the starting time (birth of TCM) up to the current time, among all the possible estimators, under some regularity conditions).

\section{E. Neuronal scale: age-dependent optimal plasticity schedul- ing}

Although the above dual optimality is described at the layer scale because of the neuronal interactions, the learning and response computation must be computed by in-place learning at each neuron. The CCI LCA [27] requires that each neuron to have a self-stored age and age-dependent plasticity schedule. Thus, every neuron in a layer has a different automatically determined (optimal) learning rate.

\section{THE FRAMEWORK OF TCM}

The brain inspired TCM is applicable to any sensory modality in principle. However, for clarity of discussion and experimental demonstration, we concentrate on text input in this paper. The temporal context with image inputs has been reported recently in Luciw et al. 2008 [43].

An English sentence $s$ is composed of a series of words: $s=\left(w_{1}, w_{2}, \ldots, w_{l}\right)$, where $l$ is the length of the sentence. The first space after a word is a word terminator, but the spaces right after the first space is treated as a space input, so that multiple spaces can be detected. All normal English punctuation marks are considered. Thus, a period is an indicator for the end of a sentence.

Unlike the "the bag of words" approach where the order of words is not considered to avoid otherwise the exponential complexity, we will use the text scan mode and reduce the exponential complexity to linear complexity. As far as we know, our system is the fist system that uses text scan mode with fully automatically generated internal representation, in contrast with, e.g., HMM based speech recognition methods which use human designed structure of multiple HMMs.

In the text scan mode, the order of the words in a sentence is important for the correctness of the sentence. The classifier is trained for a given task. In the task experimented with in this paper, is a phrase classifier. The system is trained to classify words and phrases, with equivalent words and phrases resulting in the same motor output.

The sentence classifier contains three fully connected layers, as illustrated in Fig. 1.

- Layer-1 is the input layer without computation. It has $n \times n$ input dimensions, in a square plane. Each input dimension represents a word scanned. If $n=100$, the number of neurons in the layer is $n \times n=10,000$, the size of the vocabulary.

- Layer-2 is a scan-context dependent feature matching layer. The number of neurons is $n^{2}$. When $n=64$, $n^{2}=4096$. With $k=16$ for top-k firing, the theoretical number of states that this layer can represent is at least $C_{16}^{4096} \approx 5 \times 10^{14}$, where $C_{k}^{m}$ denotes the number of k-combinations from a set of $m$ elements. It uses three sources of context input, bottom-up, lateral and top-down.

- Layer-3 is the output layer, but also serves as a layer to provide context-information for layer-2. The number of neurons in Layer 3 is equal to or larger than the number of different concepts that are necessary for a given task.

Given a sentence $s=\left(w_{1}, w_{2}, \ldots, w_{l}\right)$, the classifier scans one word at a time, learns and operates incrementally in the training phase. During the testing phase, the operation is the same except that the network (weights and neuronal ages etc.) are not updated.

Bottom-up and top-down attentive context folding: How the brain treats time has been largely a mystery [44], [45], [46]. The following cortex-inspired spatiotemporal model is a new. Consider the three-layer network in Fig. 1, running at discrete times $t=0,1,2, \ldots$.

The layer 2 takes the top-down input $\mathbf{z}(t-1)$ from layer 3 as the top-down temporal context and the bottom-up input 


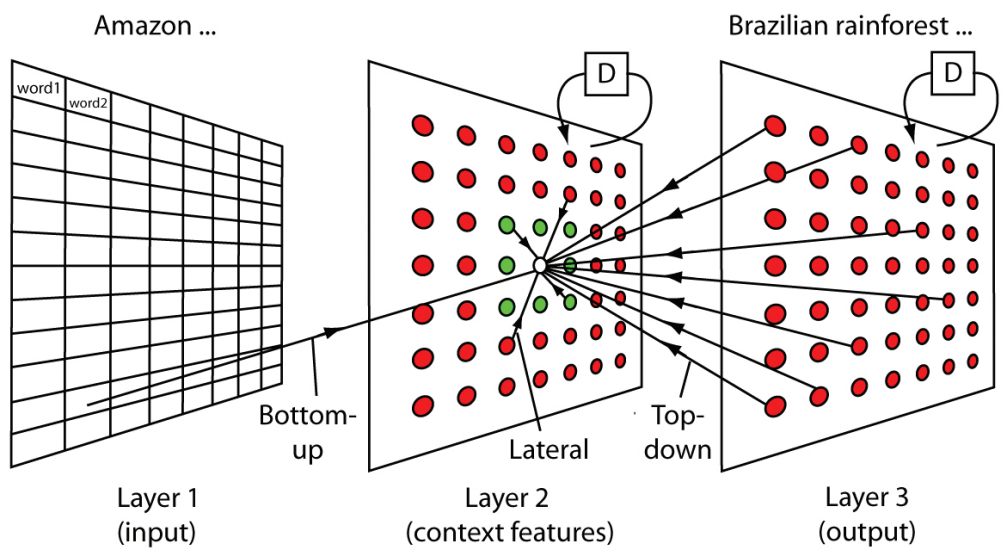

Fig. 1. The architecture of a 3-layer TCM for text processing. Only input connections to a typical neuron in layer 2 are shown. Layer 1 takes the current word input $w_{i}$. Only the component corresponding to the input word is " 1 " and all the other components are " 0 ". Layer 3 gives the current abstract context of $\left(w_{1}, w_{2}, \ldots, w_{i-1}\right)$. For sparse coding, only top-k neurons are allowed to fire and update. Each node in Layer 2 corresponds to a feature of the imbedded input space of Layer 1 and Layer 3. Some of the abstract contexts are for outputs.

$\mathbf{x}(t)$ from layer 1 which represents the current word. Its layer function implemented by LCA [27], [28] maps $\mathbf{x}(t), \mathbf{z}(t)$, based on its layer memory $L_{2}(t)$, to its response $\mathbf{y}(t+1)$ and updates the layer memory to $L_{2}(t+1)$ :

$$
\left(\mathbf{y}(t+1), L_{2}(t+1)\right)=f_{2}\left(\mathbf{x}(t), \mathbf{z}(t) \mid L_{2}(t)\right)
$$

where $f_{2}$ indicates the layer 2 function realized by LCA. Next, layer 3 takes bottom-up input $\mathbf{y}(t+1)$ from layer 2, based on its layer memory $L_{3}(t+1)$, to its response $\mathbf{z}(t+2)$ and updates the layer memory to $L_{3}(t+2)$ :

$$
\left(\mathbf{z}(t+2), L_{3}(t+2)\right)=f_{3}\left(\mathbf{y}(t+1) \mid L_{3}(t+1)\right)
$$

where $f_{3}$ indicates the layer 3 function realized by LCA. If the teacher wants to supervise the motor, impose the desired value $\mathbf{z}(t+2)$ to represent the equivalent class $\phi(\mathbf{w})$ of the attended context $\mathbf{w}$. There are several basic operations:

1) Link: If $\mathbf{z}(t+2)$ represents the context $\mathbf{z}(t)$ followed by $\mathbf{x}(t)$, the network "links" contexts to make the temporal context longer. For example, if $\mathbf{z}(t)=\phi(a b c)$ and $\mathbf{x}(t)=d$, then $\mathbf{z}(t+2)=\phi(a b c d)$, linking the class of $a b c$ with the class of $d$.

2) Drop prefix: If $\mathbf{z}(t+2)$ represents the equivalent class of $\mathbf{x}(t)$, the network "drops" the prefix. For example, if $\mathbf{z}(t)=\phi(a b c)$ and $\mathbf{x}(t)=d$, then $\mathbf{z}(t+2)=\phi(d)$, dropping the prefix $a b c$.

3) Drop postfix: If $\mathbf{z}(t+2)=\mathbf{z}(t)$, the network "drops" input $\mathbf{x}(t)$ as it keeps the last context $\mathbf{z}(t)$. For example, if $\mathbf{z}(t)=\phi(a b c)$ and $\mathbf{x}(t)=d$, then $\mathbf{z}(t+2)=\phi(a b c)$, dropping the postfix $d$.

It is then not difficult to prove that such a network of temporal abstraction is able to abstract any subset of the temporal context along the time axis and map the subset to the equivalent class at the motor output layer.

At time $t$, the network scans word $w_{t}$ and updates the net response $R(t+1)$ and the network memory $N(t+1)$ :

$$
(R(t+1), N(t+1))=f_{\mathrm{TCM}}\left(w_{t}, \mid R(t), N(t)\right) .
$$

where $f_{\mathrm{TCM}}$ indicates the function realized by the multilayer network TCM where each layer conducts LCA. After the classifier receives the first word $w_{1}$ at layer 1 , the information of the word has not reached the output layer till the network has updated twice (two time steps), because the network has two computation layers. Therefore, the network cannot produce classification result for a phrase of length $l$ until at time $l+2$ or after.

A major advantage of the proposed TCM is allowing the human teacher to supervise the motor output as the equivalent class of the attended temporal context as an output vector from layer 3 which is used not only as system output, but also used as the temporal context for the next computation.

\section{EXPERIMENTAL RESUlTS}

The architecture of the network we used in the following experiments is shown in Fig. 1. In all the experiments reported here, the training mode is supervised learning at current stage, which is to impose the correct output vector at the last layer at the desired time step. Test was done by feeding the text inputs through the network. For simplicity, we use canonical representation for text, namely a different component for a different input word or a different output label, as indicated in Fig. 1. Reinforcement learning is future work.

To show the effectiveness and efficiency of the proposed TCM, the following four tasks were learnt and evaluated. All the experiments took the size of input layer as the number of words. The hidden layer (layer 2) had $64 \times 64$ neurons, except for the task B which is $10 \times 10$. Neurons in the $3 \times 3$ neighborhoods around the winning neuron were updated to generate smooth neuronal layers. The size of the output layer was a 1-D array and equals to the number of desired outputs, which is dependent on the tasks.

Task A: New Sentences Suppose that there are four word meanings, $W_{1}, W_{2}, W_{3}, W_{4}$. Each word meaning $W_{i}$ has ten synonyms $\left\{w_{i j} \mid j=1,2, \ldots, 10\right\}, i=1,2,3,4$. Thus, there are 10000 equivalent 4-word sentences in the form of $\left(w_{1 h}, w_{2 i}, w_{3 j}, w_{4 k}\right), h, i, j, k=1,2, \ldots, 10$. How can we design the learning experiment for a developmental agent so that after learning relatively few sentences, it can recognize all the remaining new sentences that it has not learned? 
We design the lessons as follows. In Lesson 1, learn individual words:

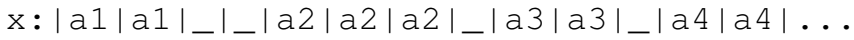
$z: I_{-}|\mathrm{A}| \mathrm{A}\left|{ }_{-}\right|_{-}|\mathrm{A}| \mathrm{A}|\mathrm{A}|_{-}|\mathrm{A}| \mathrm{A}\left|{ }_{-}\right| \mathrm{A} \mid \ldots$

where $\mathrm{x}$ is the sensory input and $\mathrm{z}$ is the corresponding motor supervision. I denotes the delimiter of a time frame. The delay in the motor output is due to that information of each sensory input takes two TCM updates to reach the motor. Do the same for $B, C$ and $D$. In Lesson 2, learn two-word sentences:

$\mathrm{x}:\left.\left.\left.|\mathrm{a} 1| \mathrm{b} 1\right|_{-}|\mathrm{a} 1| \mathrm{b} 2\right|_{-}|\mathrm{a} 1| \mathrm{b} 3\right|_{-}|\mathrm{a} 1| \mathrm{b} 4 \mid \ldots$ $z:\left.\left.\left.\left.\right|_{-}|\mathrm{A}| \mathrm{AB}\right|_{-}|\mathrm{A}| \mathrm{AB}\right|_{-}|\mathrm{A}| \mathrm{AB}\right|_{-}|\mathrm{A}| \mathrm{AB} \mid \ldots$

Only the context for the last word in each sentence is new. In Lesson 3, learn 3-word sentences in a similar way. In Lesson 4, learn 4-word sentences:

$\mathrm{x}:\left.\left.|\mathrm{a} 1| \mathrm{b} 1|\mathrm{c} 1| \mathrm{d} 1 \quad\right|_{-} \quad|\mathrm{a} 1| \mathrm{b} 1|\mathrm{C} 1| \mathrm{d} 2\right|_{-} \quad$ | . $z:\left.I_{-}|\mathrm{A}| \mathrm{AB}|\mathrm{ABC}| \mathrm{ABCD}\right|_{-}|\mathrm{A}| \mathrm{AB}|\mathrm{ABC}| \mathrm{ABCD} \mid \ldots$

The number of sentences learned in these lessons are 40, $10,10,10$, respectively. The number of new sentences to be recognized are $0,100-10,1000-10,10000-10$, respectively.

We compare TCM with MILN which classifies inputs but does not deal with time [23], [25], [43], [47]. The performances TCM are shown in Fig 2. From the results, one can see that without the temporal context, MILN cannot obtain correct outputs for all the 2-word, 3-word and 4word sentences. However, TCM achieved $\mathbf{1 0 0 \%}$ classification accuracy from epoch $23^{1}$, including 30 learned multi-word sentences and all 11070 new multi-word sentences. This is a dramatic demonstration that many new subsequences and new sequences (that have not been learned) have been perfectly mapped to the correct motor actions - the power of the recursive abstract (i.e., many to one) representation.

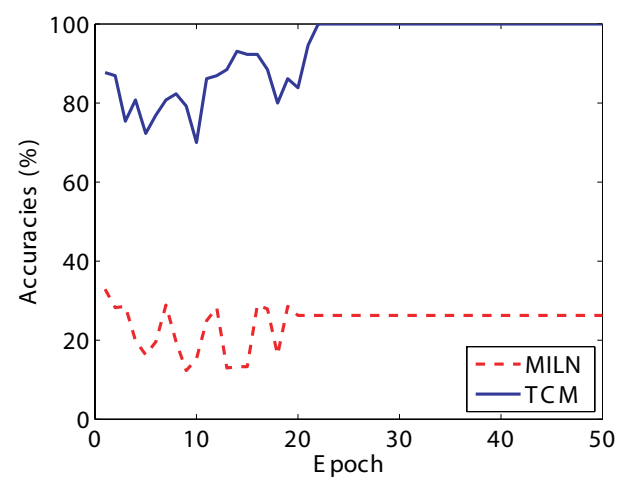

Fig. 2. Motor output accuracy versus the number of training epochs for Task A: New Sentences. All the 11070 new sentences were correctly recognized by the TCM from epoch 23 .

Task B: Word sense disambiguation (WSD) This is a task for identifying which sense of a word is used in a particular context. Usually, the context (such as neighboring words) provides an evidence for disambiguation. Without context information, the WSD task could hardly achieve an

\footnotetext{
${ }^{1}$ The brian iterates very fast, about $1 \mathrm{kHz}$, and it also needs reviews.
}

acceptable performance. For example, "Apple" represents a company name or a kind of fruit. In order to show the ability of TCM to solve this task, we build a corpus with a number of logic words. Word " $a_{1}$ ", " $a_{2}$ " and " $a_{3}$ " have one sense " $A$ ". Word " $b_{1}$ " has two senses " $B$ " and " $A B$ ". When word $b_{1}$ follows word " $a_{1}$ ", " $a_{2}$ " or " $a_{3}$ ", its sense is " $A B$ ". Words " $b_{2}$ " and " $b_{3}$ " are synonyms of " $b_{1}$ ". Word " $c_{1}$ " also has two senses " $C$ " and " $A B C$ ". When " $c_{1}$ " follows the two words "AB", the sense becomes " $A B C$ ". Based on that we built a training corpus which contains 24 instances, such as $a_{1} \rightarrow A$, $a_{1} b_{1} \rightarrow A B, a_{1} b_{2} c_{2} \rightarrow A B C$, and so on. All the other combinations (e.g. $a_{2} b_{1} c_{1}, a_{1} b_{2} c_{3}$ ) are used as test data.

In the experiment, we trained the network with the 9 distinctive words (canonical inputs) and 5 distinctive meaning outputs. The result is shown in Fig. 3. From the figure one can see that the recognition accuracies provided by TCM is much better than those by MILN that does not consider the time. In fact, TCM reached $100 \%$ recognition rate after epoch 34.

Fig. 4 shows the response of Layers 2 and 3 in the two training stages of TCM, before contexts of multiple words have been learned and after, respectively. As shown in the figure, although the same word $b_{1}$ or $c_{1}$ was received, the output at the motor layer (Layer 3) produced different actions as the TCM was taught.

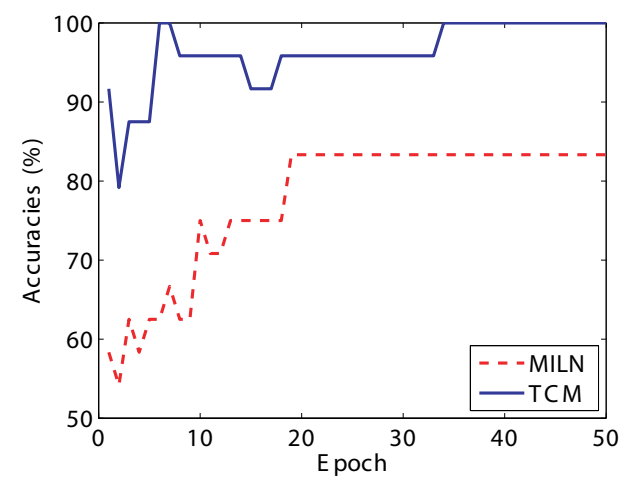

Fig. 3. Motor output accuracy versus the number of epochs through the training set for Task B: Word Sense Disambiguation.

Task C: Part-of-speech tagging. Part-of-speech (POS) tagging, a task in natural language processing, is the process of assigning the words in a sentence to the corresponding part of speech. This is an ambiguous task without temporal context, since the same word can have two different tags in two different sentences. For example:

1) This book collects images of cat.

2) The students book the tickets from this Web site.

In the first sentence, "book" is a noun, the subject of the sentence, while in the second sentence, "book" is a verb, whose subject is "The students". The correct POS tag of a word should be determined by its context.

In this experiment, we incrementally trained a TCM network to classify words into 40 POS tags according to the Penn Treebank Tag set. The corpus we used was extracted from the 


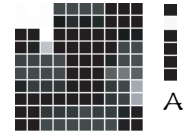

a1

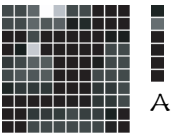

a2

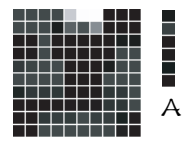

a3

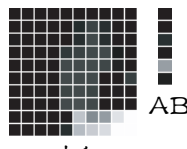

b1

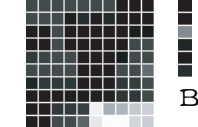

b1

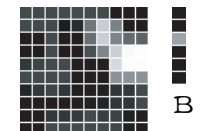

b2

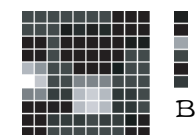

b3

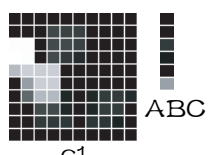

$\mathrm{C} 1$

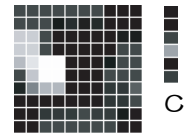

C1

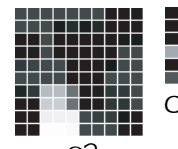

C2

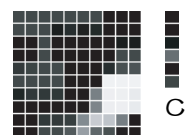

C3
Fig. 4. The responses of Layer 2 (shown as $10 \times 10$ images) and Layer 3 (shown as 6-D column vectors), when the corresponding word was presented (marked below each block). The first three rows: the responses of TCM trained with single synonyms without contexts of multiple words. The last row: the responses of TCM trained with contexts of multiple words.

CoNLL-2000 shared task ${ }^{2}$, which contains 8711 sentences and 211,727 tokens. Tagged from the Wall Street Journal corpus (WSJ), each token has been labeled POS tag and chunk tag. Limited by memory and time, the first 100 sentences were used for training and test, containing a total of 2,446 instances of words and 921 distinctive words. The size of the output layer is $1 \times 40$ (40 POS tags).

In the training phase, the network received the stream of text, one word being presented at a time sequentially, with a few space characters between every two consecutive sentences. Fig. 5 shows the accuracy after the incremental learning. With the increase of training epochs, the performances of both MILN and TCM were improved. Moreover, TCM obtained drastically better training accuracies than MILN and reached $99.6 \%$ from the 20th epoch. This shows the effectiveness of processing temporal context in TCM for a large data set. It is important to note that this is a real-time system, in the sense that the POS tag must be determined soon after a word is received, as the signal of a word flows through the pipeline of the network and reached the motor output layer. Multiple network updates were carried out at the presence of each word, so that bottom-up and top-down flows of signals can interact before the next word is presented.

Task D: Chunking. The goal of chunking is to group sequences of words together and classify them by syntactic labels. Various NLP tasks can be seen as a chunking task, such as English base noun phrase identification (base NP chunking), English base phrase identification, and so on. The chunk tag of the current token is mainly determined according to the context. For example, the sentence Mr. Carlucci served as the defense secretary in the Reagan administration. is divided into:

[NP Mr. Carlucci] [VP served] [PP as] [NP the defense secretary] [PP in] [NP the Reagan administration]. The chunk

\footnotetext{
${ }^{2}$ The corpus is available at http://www.cnts.ua.ac.be/conll2000/chunking/
}

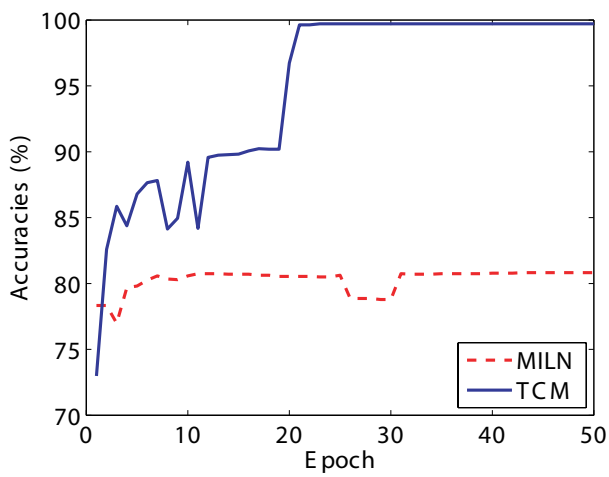

Fig. 5. Motor output accuracy versus the number of epochs for Task C: Part-of-speech Tagging.

tags are composed of the name of the chunk type and position tag, e.g., B-NP for the first word of the noun phrase words and I-VP for each of the other words in the verb phrase words. The $\mathrm{O}$ chunk tag represents tokens which do not belong to any chunk. The corpus above contains eight phrase types, such as noun phrase, verb phrase and so on. Including the $\mathrm{O}$ tag, there are a total of $8 \times 2+1=19$ chunk tags. The above example is converted into the following training format:

$\begin{array}{ll}M r . & B-N P \\ \text { Carlucci } & I-N P \\ \text { served } & B-V P \\ \text { as } & B-P P \\ \text { defense } & B-N P \\ \text { secretary } & I-N P \\ \text { in } & B-P P \\ \text { the } & B-N P \\ \text { Reagan } & I-N P \\ \text { administration } & I-N P \\ \text {. } & O\end{array}$

In the experiment, we trained the network with the top 100 sentences in the corpus used in Task C. While the size of output layer is $1 \times 19$, the input layer has 921 dimensions. The summary shown in Fig 6 indicated that the performances provided by TCM in chunking task are much better than those from MILN. TCM reached 95.2\% accuracy after epoch 18 . This task also shows that using temporal context can significantly increase the prediction accuracy. Since the outputs of some words are intrinsically ambiguous even with the temporal context, the accuracy cannot reach $100 \%$.

\section{Conclusions}

The emergent representations in existing recurrent networks for temporal processing are not abstract, limiting their power of abstract reasoning. Although it is a network, TCM is not a black box. The experimental results presented here have indicated that the Temporal Context Machines can deal with complex text processing problems with encouraging results. These experimental results are supported by a series of theoretical properties (to appear elsewhere due to space limit) of TCM. We plan to investigate a wider variety of text processing tasks and compare with traditional symbolic methods. The future work also includes applying TCM to other sensing modalities, such as video and audio streams, to investigate its power and limitations. 


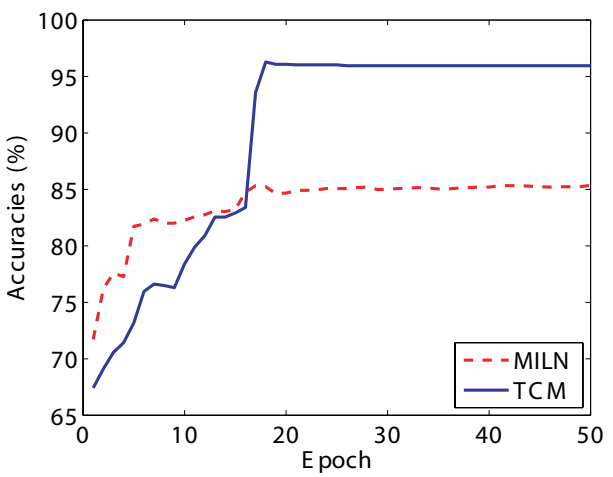

Fig. 6. Motor output accuracy versus the number of epochs for Task D: Chunking.

\section{REFERENCES}

[1] T. J. Buschman and E. K. Miller, "Top-down versus bottom-up control of attention in the prefrontal and posterior parietal cortices," Science, vol. 315, pp. 1860-1862, 2007.

[2] J. Weng, "On developmental mental architectures," Neurocomputing, vol. 70, no. 13-15, pp. 2303-2323, 2007.

[3] N. Chomsky, Rules and Representation. New York: Columbia University Press, 1978.

[4] L. R. Rabiner, "A tutorial on hidden Markov models and selected applications in speech recognition," Proceedings of IEEE, vol. 77, no. 2, pp. 257-286, 1989.

[5] _ , "Toward vision 2001: Voice and audio processing considerations," AT\&T Technical Journal, vol. 74, no. 2, pp. 4-13, 1995.

[6] J. L. Elman, E. A. Bates, M. H. Johnson, A. Karmiloff-Smith, D. Parisi, and K. Plunkett, Rethinking Innateness: A connectionist perspective on development. Cambridge, Massachusetts: MIT Press, 1997.

[7] S. Quartz and T. J. Sejnowski, "The neural basis of cognitive development: A const ructivist manifesto," Behavioral and Brain Sciences, vol. 20, no. 4, pp. 537-596, 1997.

[8] M. Sur and C. A. Leamey, "Development and plasticity of cortical areas and networks," Nature Reviews Neuroscience, vol. 2, pp. 251-262, 2001

[9] Y. Munakata and J. L. McClelland, "Connectionist models of development," Developmental Science, vol. 6, no. 4, pp. 413-429, 2003.

[10] M. Sur and J. L. R. Rubenstein, "Patterning and plasticity of the cerebral cortex," Science, vol. 310, pp. 805-810, 2005.

[11] H. Cruse, Neural Networks as Cybernetic Systems, 2nd ed. Bielefeld, Germany: Brains, Minds \& Media, 2006.

[12] J. J. Hopfield, "Neural networks and physical systems with emergent collective computational abilities," Proceedings of the National Academy of Sciences of the USA, vol. 79, no. 8, pp. 2554-2558, 1982.

[13] D. H. Ackley, G. E. hinton, and T. J. Sejnowski, "A learning algorithm for boltzmann machines," Cognitive Science, vol. 9, pp. 147-169, 1985.

[14] S. Hochreiter and J. Schmidhuber, "Long short-term memory," Neural Computation, vol. 9, no. 8, pp. 1735-1780, 1997.

[15] H. Jaeger and H. Haas, "Harnessing nonlinearity: Predicting chaotic systems and saving energy in wireless communication," Science, vol. 304, no. 5667, pp. $78-80,2004$.

[16] G. E. Hinton, P. Dayan, B. J. Frey, and R. M. Neal, "The wake-sleep algorithm for unsupervised neural networks," Science, vol. 268, pp. $1158-1161,1995$.

[17] G. E. Hinton, "Learning multiple layers of representation," Trends in Cognitive Science, vol. 11, no. 10, pp. 428-434, 2007.

[18] J. C. Wiemer, "The time-organized map algorithm: Extending the selforganizing map to spatiotemporal signals," Neural Computation, vol. 15, pp. 1143-1171, 2003.

[19] P. R. Roelfsema and A. van Ooyen, "Attention-gated reinforcement learning of internal representations for classification," Neural Computation, vol. 17, pp. 2176-2214, 2005.

[20] Y. F. Sit and R. Miikkulainen, "Self-organization of hierarchical visual maps with feedback connections," Neurocomputing, vol. 69, pp. 13091312,2006

[21] G. Golarai, D. G. Ghahremani, S. Whitfield-Gabrieli, A. Reiss, J. L. Eberhard, J. D. E. Gabrieli, and K. Grill-Spector, "Differential of high-level visual cortex correlates with category-specific recognition memory," Nature Neuroscience, vol. 10, no. 4, pp. 512-522, 2007.

[22] L. Reddy, F. Moradi, and C. Koch, "Top-down biases win against focal attention in the fusiform face area," Neuroimage, vol. 38, pp. 730-739, 2007.

[23] J. Weng, T. Luwang, H. Lu, and X. Xue, "Multilayer in-place learning networks for modeling functional layers in the laminar cortex," Neural Networks, vol. 21, pp. 150-159, 2008.

[24] Y. Zhang and J. Weng, "Task transfer by a developmental robot," IEEE Transactions on Evolutionary Computation, vol. 11, no. 2, pp. 226-248, 2007.

[25] M. Luciw and J. Weng, "Topographic class grouping with applications to $3 \mathrm{~d}$ object recognition," in IEEE World Congress on Computational Intelligence. Hong Kong: IEEE, June 1-6 2008.

[26] G. Deco and E. T. Rolls, "A neurodynamical cortical model of visual attention and invariant object recognition," Vision Research, vol. 40, pp. 2845-2859, 2004.

[27] J. Weng and N. Zhang, "Optimal in-place learning and the lobe component analysis," in Proc. IEEE World Congress on Computational Intelligence, Vancouver, BC, Canada, July 16-21 2006.

[28] J. Weng and M. Luciw, "Dually optimal neuronal layers: Lobe component analysis," IEEE Trans. Autonomous Mental Development, vol. 1, no. 1, 2009, accepted and to appear.

[29] W. K. Purves, D. Sadava, G. H. Orians, and H. C. Heller, Life: The Science of Biology, 7th ed. Sunderland, MA: Sinauer, 2004.

[30] J. H. Flavell, P. H. Miller, and S. A. Miller, Cognitive Development, 3rd ed. New Jersey: Prentice Hall, 1993.

[31] C. Blakemore and G. F. Cooper, "Development of the brain depends on the visual environment," Nature, vol. 228, pp. 477-478, Oct. 1970.

[32] M. C. Crair, D. C. Gillespie, and M. P. Stryker, "The role of visual experience in the development of columns in cat visual cortex," Science, vol. 279, pp. 566-570, 1998.

[33] D. Ansari, "Effects of development and enculturation on number representation in the brain," Nature Reviews Neuroscience, vol. 9, pp. 278291, 2008.

[34] J. Weng, N. Ahuja, and T. S. Huang, "Learning recognition and segmentation using the Cresceptron," International Journal of Computer Vision, vol. 25, no. 2, pp. 109-143, Nov. 1997.

[35] N. Almassy, G. M. Edelman, and O. Sporns, "Behavioral constraints in the development of neural properties: A cortical model embedded in a real-world device," Cerebral Cortex, vol. 8, no. 4, pp. 346-361, 1998.

[36] J. Weng, J. McClelland, A. Pentland, O. Sporns, I. Stockman, M. Sur, and E. Thelen, "Autonomous mental development by robots and animals," Science, vol. 291, no. 5504, pp. 599-600, 2001.

[37] J. Weng and W. Hwang, "From neural networks to the brain: Autonomous mental development," IEEE Computational Intelligence Magazine, vol. 1, no. 3, pp. 15-31, 2006.

[38] D. J. Felleman and D. C. Van Essen, "Distributed hierarchical processing in the primate cerebral cortex," Cerebral Cortex, vol. 1, pp. 1-47, 1991.

[39] E. M. Callaway, "Local circuits in primary visual cortex of the macaque monkey," Annual Review of Neuroscience, vol. 21, pp. 47-74, 1998.

[40] A. K. Wiser and E. M. Callaway, "Contributions of individual layer 6 pyramidal neurons to local circuitry in macaque primary visual cortex," Journal of neuroscience, vol. 16, pp. 2724-2739, 1996.

[41] S. Grossberg and R. Raizada, "Contrast-sensitive perceptual grouping and object-based attention in the laminar circuits of primary visual cortex," Vision Research, vol. 40, pp. 1413-1432, 2000.

[42] B. A. Olshaushen and D. J. Field, "Emergence of simple-cell receptive field properties by learning a sparse code for natural images," Nature, vol. 381, pp. 607-609, June 131996.

[43] M. Luciw, J. Weng, and S. Zeng, "Motor initiated expectation through top-down connections as abstract context in a physical world," in IEEE International Conference on Development and Learning, Monterey, CA, Aug. 9-12 2008.

[44] M. D. Mauk and D. V. Buonomano, "The neural basis of temporal processing," Annual Review of Neuroscience, vol. 27, pp. 307-340, 2004.

[45] P. J. Drew and L. F. Abbott, "Extending the effects of spike-timingdependent plasticity to behavioral timescales," The National Academy of Sciences of the USA, vol. 103, no. 23, pp. 8876-8881, 2006.

[46] I. Ito, R. C. Ong, B. Raman, and M. Stopfer, "Sparse odor representation and olfactory learning," Nature Neuroscience, vol. 11, no. 10, pp. 11771184, 2008.

[47] Z. Ji, J. Weng, and D. Prokhorov, "Where-what network 1: "Where" and "What" assist each other through top-down connections," in Proc. IEEE International Conference on Development and Learning, Monterey, CA, Aug. 9-12 2008. 